\title{
Computer-assisted orthopedic surgery research and implementation of measurement techniques
}

\author{
Zhao Ming, Fan Xincan \\ School of computer engineering, Shenzhen Polytechnic, Shenzhen 518055, China \\ zhaoming@szpt.edu.cn, horsefxc@szpt.edu.cn
}

Keywords: TKA; 3-dimensional localization;3-dimention reconstruction; biometric measurement

\begin{abstract}
This paper studies the key technologies of the computer-aided bone surgery system testing techniques, and mathematical modeling, the combination of computer image processing, built knee replacement surgery in the lower extremity biomechanics line measurement system and validated by experiments.
\end{abstract}

\section{Introduction}

According to statistics, half of the population over the age of 50 suffers from osteoarthritis; $90 \%$ of women and $80 \%$ of men in the population over the age of 65 suffer from osteoarthritis. While there is a lot less than traditional orthopedic surgery, such as three-dimensional reconstruction is not intuitive, difficult navigation positioning measurement techniques to determine many of the urgent need to address the problem of cutting plane difficult. Through the use of digital medical imaging technology and space positioning technology can significantly improve the quality of surgery to improve the environment of the patient's recovery. Domestic research focuses on computer medical imaging technology in medical image processing and recognition; there is a considerable gap between the image navigation system with foreign.

The computer-assisted orthopedic surgery measurement system is applied to the lower extremity biomechanics line of total knee surgery and soft tissue balance measurement in computer image processing, spatial orientation tracking technology. Measurement techniques of computer-assisted orthopedic surgery research in the field of orthopedics first CT image reconstruction assisted pedicle screw implantation, later to be further used for hip and knee replacement. More than a decade, the technology development is extremely rapid, and now has been carried out in almost all areas of orthopedic clinical practice, and achieved initial results. The specific scope of the hip replacement, total knee replacement in spinal surgery, arthroscopic surgery, including fixture placement $^{[1]}$.

\section{Technology}

For surgical navigation system in total knee arthroplasty (TKA) surgery needs further development targeted aided measurement system in the main navigation system, the main lines of force TKA surgery on-site measurement and adjustment by navigation technology to improve the biomechanical properties of the TKA surgery.

System using mathematical modeling, computer image processing combined analog measurement of the related technologies in the lower limb simulation model, the technology to achieve the following key points ${ }^{[2]}$ :

(1) Space for three-dimensional positioning system data read

The system uses the NDI Company Polaris system, which is based on optical positioning sensor CCD camera as the measurement objective is to install several infrared light - emitting diodes on the surgical instruments. The spatial position through the infrared light-emitting diode, the calculated position and attitude of the surgical instrument.

(2) The image processing and image display

The system software using VC + + programming, application VTK package complete image 
processing and 3D reconstruction, and the QT development package to complete the preparation interface. Completed two additional projection plane based on the DICOM medical image reading, the operation requirement under the clinician, on the basis of the original image (cross-section), through the image interpolation method reconstruction of three-dimensional image, sagittal and coronal anatomy.

(3) Registration

Coordinate system of the world coordinate (in kind), image coordinate system (CT or MRI images), the instrument position measurement coordinate transformation between the coordinate system (GPS).

(4) Biometrics technology

Location and tracking system, the measurement of geometric parameters of the knee, completed the three-dimensional image reconstruction the hip center ankle center and three-dimensional coordinates of the center of the knee.

\section{System Design}

System implementations using mathematical modeling, computer image processing combined analog measurement of the related technologies in the lower limb simulation model. The system design shown in Figure 1:

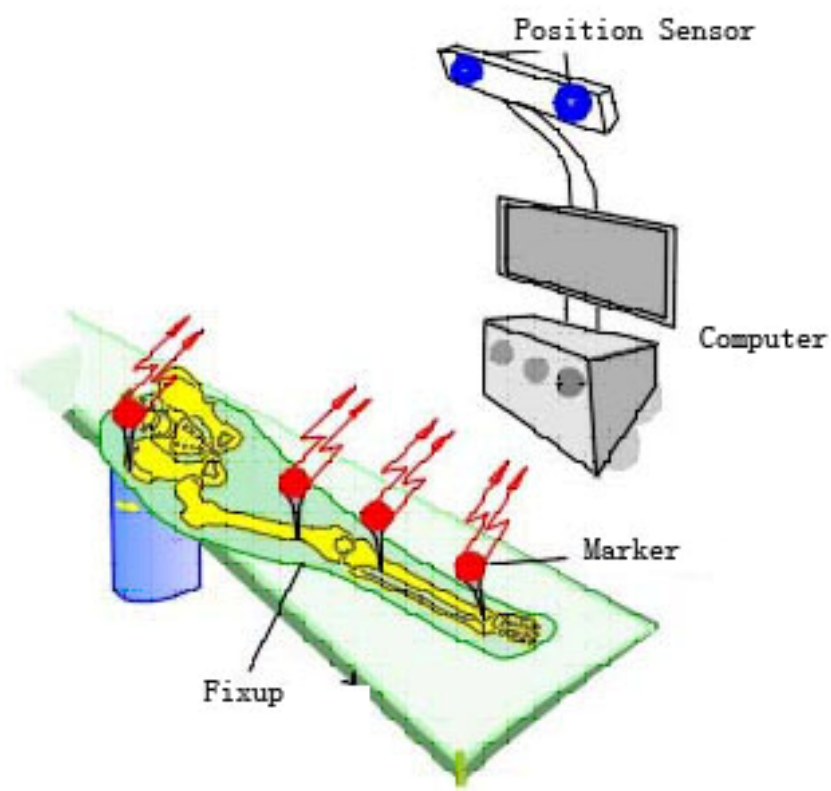

Figure 1 Computer-aided measurement system diagram of lower extremity biomechanics line

(1) space for three-dimensional positioning system data read

Computer-aided navigation system, spatial orientation is the key to the whole system, is directly related to the success or failure of the entire system's accuracy and computer-assisted surgery, its role is measured in real time space position and attitude of the surgical instruments. Depending on the positioning sensor, can be divided into mechanical positioning ultrasonic positioning electromagnetic positioning and optical positioning method. The system uses the NDI Company Polaris system, which is based on optical positioning sensor CCD camera as the measurement objective is to install several infrared light - emitting diodes on the surgical instruments. The spatial position through the infrared light-emitting diode, the calculated position and attitude of the surgical instrument $^{[3]}$.

(2) The image processing and image display

Two additional projection plane based on the DICOM medical image is read, according to the the clinician operating requirements, on the basis of the original image (cross-section), through methods such as image interpolation reconstructed three-dimensional image, sagittal and coronal plane of the 
anatomical Figure.

After Get sequence DICOM image by the surface rendering three-dimensional image reconstruction method, draw out the femur, tibia, and three-dimensional surface of the stereoscopic $\operatorname{image}^{[4]}$.

(3) System Registration

CT images are taken before surgery, and the surgical procedure, the system needs to be done for linking the position of the surgical instrument and a CT image, and the actual shares tibia alignment. In a total of three coordinate system: world coordinate system, the image coordinate system, instrument position measurement coordinate system. The alignment process of the establishment process, contact between these coordinates Once the transformation relationship between the coordinate system is determined, it can be easily measured location of the instrument in the coordinate system is transformed into the image coordinate system is displayed ${ }^{[5]}$.

Alignment of the basic principle: select some suitable feature point, the coordinates of these characteristic points in the three coordinates were measured, and the transformation relationship between the coordinate system is determined by the matrix transformation. Wherein the coordinates of the feature points in the image coordinate system can be obtained by image processing, the coordinates in the measurement coordinate system can click on the feature points to the surgical instrument to get.

(4) Biological lines of force measuring geometric parameters

Biological lines of force measurement is to use the navigation technology to measure hip center, the three-dimensional position of the center of the ankle and knee center. Obtain a series of points of the three-dimensional coordinates, and then use these points to solve the femoral head in the center of the sphere, to thereby obtain the center of the hip joint by the tracking device fixed on the femoral shaft, rotation of the femoral ${ }^{[6]}$. The position of the ankle joint center can also be carried out by a similar method. The approximate coincidence anatomical lines of force of the tibia and biological lines of force by anatomical features to determine the three-dimensional position of the center of the ankle. Femoral shaft center to mark the knee joint center to determine navigation system to measure the hip joint center and ankle center connection, the line connecting the center of the hip joint center and knee, and the line connecting the center of the knee joint center and ankle. These three lines do not coincide, due to distortion, according to the positional relationship of these three lines, to adjust the position of the biological lines of force, the biological line of force to return to its normal position ${ }^{[7]}$.

Due to the deformation of the biological lines of force, resulting in damage to the knee joint surfaces, and joint surgery is the removal of the damaged particular surface, according to the deformation of the biological lines of force, and measuring the position of the breakage condyle surface, determining the position and orientation of the cutting plane.

\section{Experiments and analysis}

System experimental platform is shown in Figure 2, through the use of $\mathrm{VC}++$ programming, application VTK package complete image processing and 3D reconstruction, and the QT development package to complete the preparation interface; operation interface design based on the QT development package;VTK package, complete reading based on the DICOM medical image, and reconstruct the three-dimensional image ${ }^{[8]}$. 


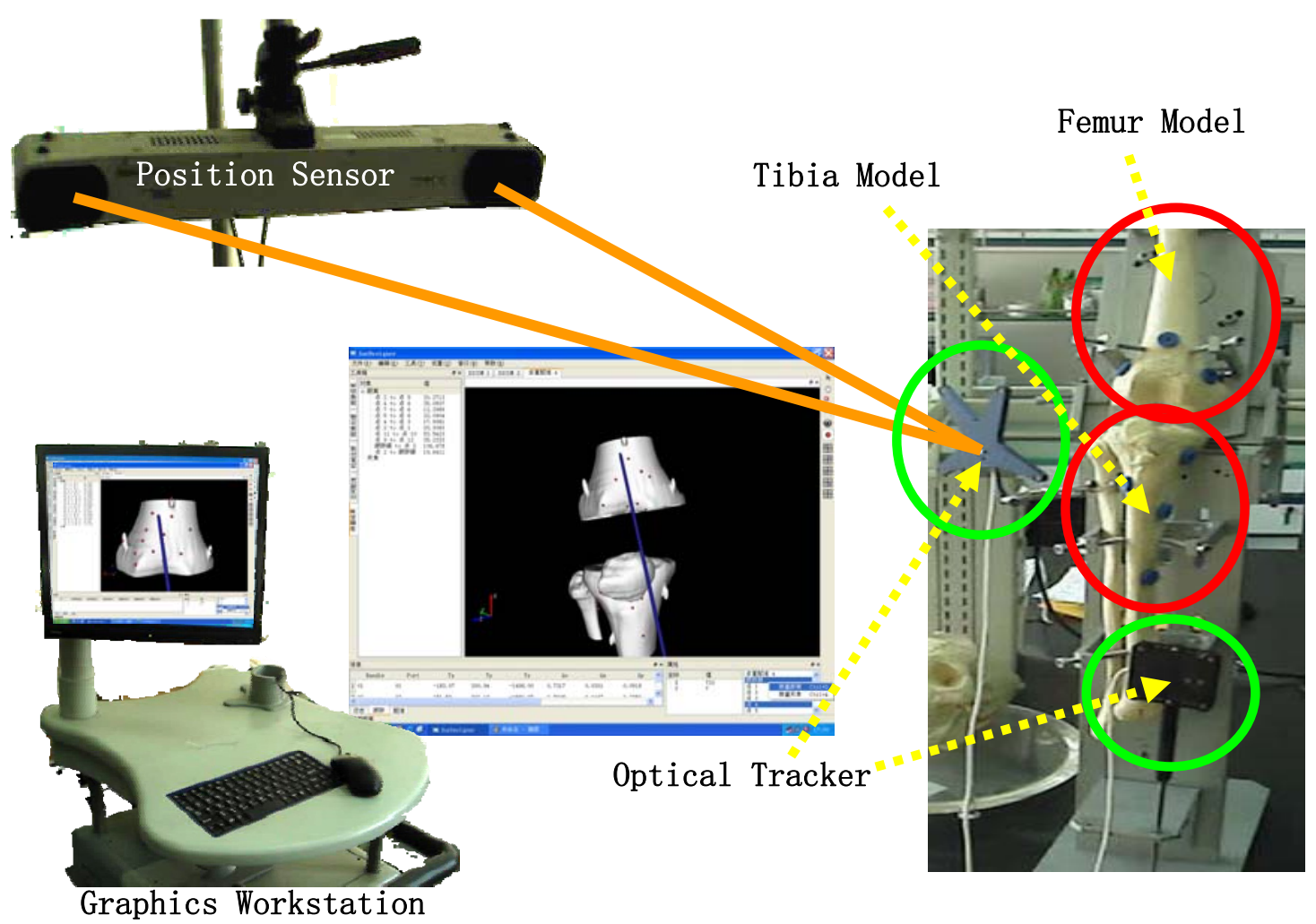

Figure 2 system experimental platforms

The experimental procedure is divided into the following steps ${ }^{[9]}$ :

- The sequence read DICOM images

- image processing and display

- three-dimensional reconstruction

- System Registration

- real-time navigation

- The point of measurement

- distance, angle, line of force measurement

The experiments show that:

(1) The application of three-dimensional reconstruction techniques, surgery is more intuitive.

VTK package, read the medical image based on the DICOM protocol, and according to the clinician operating requirements, on the basis of the original image (cross-section), through the image interpolation method reconstruct the other two of the three-dimensional image plane of projection, the vector like and coronal planes.

After Get sequence DICOM image by the surface rendering three-dimensional image reconstruction method, draw out the femur, tibia, and three-dimensional surface of the stereoscopic image.

(2) The measurement of biological lines of force to make surgery more precise.

No image to determine the center of the hip, and ankle joint centers and the three-dimensional position of the center of the knee joint.

Calculation of total knee replacement surgery in biological lines of force measurement, and on this basis to determine the position and orientation of the cutting plane of the particular surface of the broken bone.

(3) The application of surgical navigation technology minimally invasive, make patients safer.

The real-time optical 3D measurement systems that take advantage of the Canada the NDI Company's Polaris series the Polaris P4 optical tracking system to track the total knee replacement 
surgery.

\section{References}

[1] Wu Jian, Ye Datian, Development of the technique for measuring soft tissue balance in knee replacement, Journal of Biomedical Engineering, 2007; 24(4): 937-940

[2] Jian Wu, You Wang, Kerong Dai, et al A method for dynamically measuring the Soft Tissue Balance in the Total Knee Replacement, World Congress on Medical Physics and Biomedical Engineering 2006, Seoul, 2006.8

[3] C. Marmignon, A. Leimnei, P. Cinquin et al. A Computer-assisted controlled distraction device to guide ligament balancing during knee arthroplasty, CAOS 2004; 65-66

[4] C. Mahr, M. Gonzalez, Farid Amirouche, et al. A Real-TimeDynamic Contact Pressure Device for Tibia Trials in TKA, 2003.4

[5] Pei Fuxing, Li Cheng computer navigation in total knee replacement system [J]. The China orthopedic the magazine .2006,26 (10),699-702

[6] Guokai today Chengzhao Ming, Lei. Knee arthroplasty tibia prosthesis point, line, surface placed knee function recovery relationship [J]. Chinese Journal of Clinical Rehabilitation, 2005,9 (2) :220-222.

[7] Feng Guozhang, wearing No. money extraordinary. Infrared navigation total knee replacement surgery dominant performance assessment [J]. Spine, 2004, 24 (9):572-574.

[8] $\mathrm{Xu}$ Jie, Ma, if where, Ding Yue. Knee replacement arthroplasty assisted positioning device three-dimensional navigation dominant performance assessment [J]. Chinese Journal of Clinical Rehabilitation, 2006, 10 (1):106-108.

[9] Chang Wei, horses, such as word, Henan vibration word. Wang Tao., Like registration applications in computer-assisted total knee replacement surgery [J] Computer Engineering .2002, 28 (5), 225-226 